\title{
Reconciling resummation and renormalization
}

\author{
Antal Jakovac* \\ BME, Institute of Physics, Budafoki út 8, H-1111 Budapest, Hungary \\ E-mail: Antal.Jakovacecern.ch
}

In the naive form of most resummations we get into conflict with order-by-order renormalization. We present a method that is capable to ensure UV consistency of any resummations satisfying certain conditions. The method is based on the observation that resummation is equivalent with a calculation in an adequate perturbation scheme, followed by a renormalization scheme changing. This framework works both in static and momentum-dependent cases. In particular it is possible to establish finite infrared 2PI resummation.

29th Johns Hopkins Workshop on current problems in particle theory: strong matter in the heavens 1-3 August

Budapest

${ }^{*}$ Speaker. 


\section{Introduction}

In a theory with small coupling constants we expect that perturbation theory works well; in fact what we understand under this sentence is that renormalized perturbation theory with some standard renormalizations scheme (eg. $\overline{\mathrm{MS}}$ or on-mass-shell scheme) provides small corrections as compared to the leading order. When this approach fails, we tend to speak about the failure of perturbation theory itself. This failure can sometimes be associated to certain class of diagrams in the chosen scheme. Then we have the hope that after resummation of this subset of diagrams we can still give analytic predictions for the observable in question. We can give several examples when we need resummation: self-energy resummations (Schwinger-Dyson equation) is needed to determine mass shift, daisy resummation [1] is needed at high temperatures in a scalar field theory, HTL resummation [2] should be used in gauge theories at high temperatures. In 2PI and higher point irreducible resummation we can work with exact propagators/vertices [3]. And also the renormalization group flow defines a set of resummed theories parameterized by the renormalization scale [4].

In most cases resummations are designed to solve some infrared (IR) problems, ie. it resums diagrams that are the most sensitive in the IR regime. The corresponding counterterm diagrams that are needed to ensure finiteness of the perturbation theory at high momenta, are often neglected. This has a consequence that ultraviolet (UV) consistency becomes a serious problem in the resummed theories. The problem becomes even deeper in case of momentum dependent resummations, like the 2PI resummation. There are different approaches published recently to overcome this difficulty, primarily in the static and 2PI resummation case [5].

In the present paper we try to describe an approach different from the above, mainly diagrammatic methods. The idea is that the only reliable analytic method to treat UV divergences is the renormalized perturbation theory [4]. In some way all the consistent resummations have to be linked to a specific scheme. The task is to find this link for each specific resummation method.

To understand the relation between the resummation methods and renormalization schemes we recall that renormalized perturbation theory has a large freedom in choosing the finite parts of the counterterms. We can use this freedom to choose finite parts such a way that mostly reduces the IR sensitivity of the system. The so-defined scheme will depend on the environmental parameters (like temperature). In order to have results in a reference scheme (eg. $\overline{\mathrm{MS}}$ ) we must perform a matching between the parameters of the perturbation scheme and the reference scheme, using the requirement that the bare Lagrangian are the same. Both being renormalization schemes, this is sufficient to match all of the observables to the given order in perturbation theory [4]. The difference of the higher order terms is the resummation. The idea of fitting the perturbation theory to the environment was used already in [6] where the authors tuned the renormalization scale appropriately.

This strategy will be described in this contribution in more detail. First we examine the problem in the simplest static mass resummation case, and we show how the method sketched above will solve the UV consistency problem, in principle and in the $\Phi^{4}$ theory. For a more elaborated description of these section cf. [7]. Then we change to the momentum dependent case, where basically the same method works, but we have to take care some details. As a special application we present how a 2PI resummation can be represented by a scheme, and how can it solve the 
renormalizability problem. Finally we give a short summary of the method.

\section{Thermal mass resummation - the naive method}

A nice and well-known example of a system that needs resummation is the high temperature $\Phi^{4}$ theory. In this model, as was shown by Dolan and Jackiw [1], at $n$-th order of perturbation theory we obtain a mass correction of order $\lambda T^{2}\left(\lambda T^{2} / m^{2}\right)^{n-1}$, which is a growing function of the coupling constant if $T \gg m$. In this case the class of daisy diagrams was the adequate subset which was needed to sum up. To facilitate the treatment, the same effect could have been achieved with the thermal counterterm method [8], where we subtracted from and added to the Lagrangian the same term, but treating them at different loop order. In this way it was possible to change the tree level mass without the change of the Lagrangian. If we denote the free mass-squared by $m^{2}$ and the resummed mass by $M_{T}^{2}=m^{2}+\Delta M_{T}^{2}$, then the mass terms of the Lagrangian will be written as

$$
-\mathscr{L}_{\text {mass }}=\underbrace{\frac{M_{T}^{2}}{2} \varphi^{2}}_{\text {tree level }}+\underbrace{\left(-\frac{M_{T}^{2}-m^{2}}{2} \varphi^{2}+\frac{\delta m^{2}}{2} \varphi^{2}\right)}_{\text {one loop }} .
$$

To determine the ideal value of the added-subtracted mass one should use an additional requirement, for example that the one-loop level mass term is just $M_{T}^{2}$. Denoting the complete unresummed one-loop self energy at momentum $k$ and with $M^{2}$ mass-squared on the internal lines by $\Pi\left(k ; M^{2}\right)$ we find

$$
M_{T}^{2}=m^{2}+\Pi\left(k=0, M_{T}^{2}\right) .
$$

This is an implicit (gap) equation for the mass $M_{T}^{2}$ as a function of $m^{2}$. In other models, or for other infrared (IR) problems other type of resummations proved to be useful (like super-daisy, HTL, 2PI, RG etc.)

This appealing method has, however, a severe drawback: taking it really seriously it provides ultraviolet (UV) divergent result. The symbol $\Pi$ in (2.2), in fact has contributions from the tadpole diagram and the mass counterterm. The tadpole contribution, according to (2.2), has to be computed with internal propagators with $M_{T}^{2}$ mass-squared; it reads

$$
T_{B}\left(M_{T}^{2}\right)=\frac{M_{T}^{2}}{16 \pi^{2}}\left[-\frac{1}{\varepsilon}+\gamma_{E}-1+\ln \frac{M_{T}^{2}}{4 \pi \mu^{2}}\right]+\frac{1}{2 \pi^{2}} \int_{M}^{\infty} d \omega \sqrt{\omega^{2}-M_{T}^{2}} n(\omega) .
$$

The mass counterterm, on the other hand, has a value fixed by the renormalization scheme; if we use $\overline{\mathrm{MS}}$ then we have

$$
\delta m^{2}=-\frac{\lambda m^{2}}{32 \pi^{2}}\left[-\frac{1}{\varepsilon}+\gamma_{E}-1+\ln \frac{M_{T}^{2}}{4 \pi \mu^{2}}\right]
$$

The self-energy therefore

$$
\Pi\left(k=0, M_{T}^{2}\right)=\frac{\lambda}{2} T_{B}\left(M_{T}^{2}\right)+\delta m^{2}=-\frac{\lambda\left(M_{T}^{2}-m^{2}\right)}{32 \pi^{2}} \frac{1}{\varepsilon}+\ldots
$$

is UV divergent. 


\section{Treating UV divergences in perturbation theory}

The main reason for this divergence is that the finiteness of perturbation theory depends on a very sensitive balance between the counterterms and 2PI diagrams [4]. In fact only a small subclass of all conceivable perturbation theories can fulfill all the requirements to provide order-by-order finiteness: these are the renormalized perturbation series of a renormalizable theory. So when UV finiteness is a crucial issue, then we must remain within this subclass, we must use renormalized perturbation theory for resummation, too.

Fortunately this subclass is rather wide, as we can freely choose the finite part of all the counterterms at all orders. All choices yield different renormalized perturbation series; ie. they provide different results at any fixed order as a function of the renormalized parameters of the Lagrangian. One is common in all of these results: they are all finite. These span the space of "perturbatively reachable" domain in a given theory. We should speak about "non-perturbative effect" only if some phenomenon lies outside of this domain.

As the example of the thermal mass resummation indicated, a generic perturbation theory has a very small convergence radius (in the weak "asymptotic convergence" sense) because of IR sensitivity. Only schemes well adapted to the environment [6] show good convergence properties. To give a well-known example: in the $\Phi^{4}$ theory with negative mass-squared we do not work in the original free Hilbert space, since there the masses are imaginary, instead we adapt the perturbation theory to the (expected) vacuum properties and use a Hilbert space built on the spontaneously broken vacuum.

Usually we want to compare result coming from different environments: for example we want to know the thermal properties as a function of the zero temperature observables. Different environments need different schemes, whose results are not directly comparable. This is because different choices of the finite parts formally yield different bare Lagrangian and so different physics. But the bare Lagrangian is completely determined by the bare parameters (as bare masses, couplings, wave function renormalizations), so if we require that the bare parameters are equal we can hold the physics constant in the two schemes. Expressing as function of renormalized parameters in each specific schemes, the equality of bare parameters impose relations between the renormalized parameters of different schemes. For example in $\Phi^{4}$ theory, where we have 3 renormalized parameters ( $Z$ wave function renormalization constant, $m^{2}$ mass-squared and $\lambda$ coupling constant), we will have the following relations

$$
Z_{A}=Z_{B}, \quad m_{A}^{2}+\delta m_{A}^{2}=m_{B}^{2}+\delta m_{B}^{2}, \quad \lambda_{A}+\delta \lambda_{A}=\lambda_{B}+\delta \lambda_{B} .
$$

If two schemes are related in this way, then there different results for a given observable can be considered as a resummation effect, since at infinite loop order they both yield the same, exact result.

\section{Example: thermal mass resummation in $\Phi^{4}$ theory}

Using the ideas above we can work out the thermal mass resummation in a consistent way. In any perturbation schemes the one-loop self-energy reads

$$
\Pi(k=0)=\frac{\lambda}{2} T_{B}\left(m^{2}\right)+\delta m_{1}^{2},
$$


where $T_{B}$ was defined in (2.3). In $\overline{\mathrm{MS}}$ scheme we choose (2.4) for the mass counterterm. However, only the divergent part is really fixed by the condition of renormalizability, different schemes can use different finite parts. A specific choice can be:

$$
\delta m_{1, \mathrm{res}}^{2}=-\frac{\lambda}{2} T_{B}\left(m^{2}\right)
$$

This scheme depend on the temperature, but only through the finite parts. At one hand this is allowed mathematically, on the other hand this is expected: the environment, we have to be adapted to, is represented by the temperature. In this res scheme the one loop self energy is zero

$$
\Pi_{\text {res }}(k=0)=0,
$$

so it is in fact a finite temperature mass-shell scheme: the complete self energy is just the mass.

If we use $\overline{\mathrm{MS}}$ at zero temperature and res scheme at finite temperature we have to ensure that they describe the same physics, ie. they stem from the same bare Lagrangian. In this simple example this requirement reduces a relation between the renormalized mass values in the two schemes. Expressing the bare mass to order $\lambda$ in both cases, we find

$$
Z^{2} m_{\text {bare }}^{2}=m_{\mathrm{res}}^{2}+\delta m_{1, \mathrm{res}}^{2}=m_{\overline{\mathrm{MS}}}^{2}+\delta m_{\overline{\mathrm{MS}}}^{2} \quad \Rightarrow \quad m_{\mathrm{MS}}^{2}=m_{\mathrm{res}}^{2}-T_{B, \overline{\mathrm{MS}}}\left(m_{\mathrm{res}}^{2}\right),
$$

where the last symbol means

$$
T_{B, \overline{\mathrm{MS}}}\left(m_{\mathrm{res}}^{2}\right)=\frac{\lambda m_{\mathrm{res}}^{2}}{32 \pi^{2}} \ln \frac{m_{\mathrm{res}}^{2}}{\mu^{2}}+\frac{\lambda}{4 \pi^{2}} \int_{m_{\mathrm{res}}}^{\infty} d \omega \sqrt{\omega^{2}-m_{\mathrm{res}}^{2}} n(\omega),
$$

which is nothing but the tadpole diagram renormalized in $\overline{\mathrm{MS}}$ scheme, evaluated at $m^{2}=m_{\mathrm{res}}^{2}$ point. So, in fact, the gap equation (2.2) is true in the sense that $\Pi$ is the renormalized self energy correction.

\section{Momentum dependence}

What was said so far applies for the momentum-independent resummation. In many cases, however, this is not enough to fully diminish IR sensitivity from the system. In these cases we have to apply momentum dependent resummations.

The way we have adapted the renormalization scheme to the environment was the proper choice of finite parts of the counterterms. Momentum dependent resummations therefore imply usage of momentum dependent finite parts. Formally this is feasible, the only question is that whether in this way we do not spoil renormalizability.

Let us concentrate on the mass resummation in this proceedings, the more elaborated complete discussion will be published elsewhere [9]. We now choose $\delta m^{2}(k)$, momentum dependent counterterm. In this case the matching to a reference scheme, eg. $\overline{\mathrm{MS}}$, yields the condition

$$
Z^{2} m_{\text {bare }}^{2}=m_{\overline{\mathrm{MS}}}^{2}+\delta m_{\mathrm{MS}}^{2}=m^{2}+\delta m^{2}(k) .
$$

This enforces to work with momentum dependent tree level mass from the beginning, ie. $m^{2}=$ $m^{2}(k)$. 
We can maintain renormalizability, provided we satisfy some requirements. First the divergent part of the counterterm should be unique, ie. the same as, say, for the $\overline{\mathrm{MS}}$ case. Secondly, the momentum dependence of the tree level mass should not generate new divergences. We will assume that for asymptotically large momentum the tree level mass behaves as

$$
m^{2}(k)=m_{R}^{2}+\mathscr{O}\left(k^{-\gamma}\right) .
$$

The most singular diagram is the tadpole. If there are no new divergences in the tadpole, then there are no new divergences in any other diagrams. With momentum dependent mass the value of the tadpole reads

$$
\begin{aligned}
\int \frac{d^{4} p}{(2 \pi)^{4}} \frac{1}{p^{2}-m^{2}(p)} & =\int \frac{d^{4} p}{(2 \pi)^{4}} \frac{1}{p^{2}-m_{R}^{2}-\mathscr{O}\left(p^{-\gamma}\right)}= \\
& =\int \frac{d^{4} p}{(2 \pi)^{4}}\left[\frac{1}{p^{2}-m_{R}^{2}}+\mathscr{O}\left(\frac{p^{-\gamma}}{\left(p^{2}-m_{R}^{2}\right)^{2}}\right)\right] .
\end{aligned}
$$

The first term is the usual tadpole contribution. The second term is finite if $\gamma>0$. So if the momentum dependent mass approaches its limiting value as a power law, then the divergence structure remain untouched.

\section{The $2 \mathrm{PI}$ resummation}

Let us write the self energy as

$$
\Pi(k)=\delta m^{2}+\bar{\Pi}\left(k, m^{2}\right) .
$$

In the mass-shell scheme we choose $\delta m^{2}=-\bar{\Pi}\left(k=m^{2}\right)$ at zero temperature. We have seen that if we apply the same prescription at finite temperature, it leads to thermal mass resummation. Going on with this idea we may try to choose

$$
\delta m^{2}(k)=-\bar{\Pi}\left(k, m^{2}(k)\right)
$$

at any momenta. This has the consequence that $\Pi(k)=0$, ie. there is no self-energy correction whatsoever in this scheme! As a resummation, therefore, it provides self-energy correction resummation (also in the internal lines!), which is just the 2PI resummation. So we will call this scheme as 2PI scheme.

If we compare with the $\overline{\mathrm{MS}}$ scheme the requirement of the constant physics reads

$$
m^{2}(k)-\bar{\Pi}\left(k, m^{2}(k)\right)=m_{\overline{\mathrm{MS}}}^{2}+\delta m_{\overline{\mathrm{MS}}}^{2} .
$$

Since the divergence structure of $-\bar{\Pi}\left(k, m^{2}(k)\right)$ is the same as that of $\delta m_{\overline{\mathrm{MS}}}^{2}$ the expression $\bar{\Pi}\left(k, m^{2}(k)\right)+$ $\delta m_{\mathrm{MS}}^{2}$ is finite, and diagrammatically it contains no self-energy correction in the internal lines; therefore we will call it $\bar{\Pi}_{2 P I}$ since its meaning is really the self-energy renormalized in the 2PI scheme.

In the language of the propagator $G^{-1}(k)=k^{2}-m^{2}$ the above relation can be written as

$$
G_{2 P I}^{-1}(k)=G_{\overline{\mathrm{MS}}}^{-1}(k)+\bar{\Pi}_{2 P I}\left(k, G_{2 P I}(k)\right) .
$$


If we identify $G_{\overline{\mathrm{MS}}}$ as the "free propagator" and $G_{2 P I}$ as the "resummed propagator", then this equation is exactly the basic relation of the 2PI resummation [3].

But in the light of renormalizability the above procedure is not satisfactory. In the perturbative evaluation of $\bar{\Pi}(k)$ we will encounter terms like $k^{2} \ln k$ or $m^{2} \ln k$, which are not allowed contributions to $\delta m^{2}(k)$. Therefore the naive 2PI approach is not renormalizable - as it is well known in the 2PI literature [3].

It is possible to give a solution to the problem of 2PI renormalizability in the present context. We are not enforced now to choose exactly the $\bar{\Pi}$ self energy; after all, resummation is needed by IR sensitivity, ie. a low momenta phenomenon. It is enough to perform $2 \mathrm{PI}$ resummation in this domain, too. We can, therefore, simply omit the terms that high momentum terms that would violate renormalizability. We can do it with some (smooth) cutoff function $\Theta(x)$, and choose, for example

$$
\delta m^{2}(k)=-\Theta(k / \Lambda) \bar{\Pi}\left(k, m^{2}(k)\right)-(1-\Theta(k / \Lambda)) \delta m_{\overline{\mathrm{MS}}}^{2} .
$$

For another solution we realize that since the problem comes from asymptotically large momenta this is insensitive to the environment; in particular they appear in the same way at zero temperature, and in $\overline{\mathrm{MS}}$ scheme. If we subtract the zero temperature perturbative self energy renormalized in $\overline{\mathrm{MS}}$, the rest is already appropriate to play the role of a momentum dependent mass counterterm. We should be careful, however, since asymptotically divergent contributions may come from subdiagrams, and so the subtraction has to be repeated to every sub-diagram, in the spirit of the forest formula. After these subtractions we can write

$$
\delta m^{2}(k)=-\bar{\Pi}_{\text {asympt. subtr. }}\left(k, m^{2}(k)\right) .
$$

\section{Summary}

To summarize the content of this contribution we repeat that we must not resum IR sensitive diagrams without respect of the UV consistency, otherwise we run into divergences even in the most simple cases. The perturbative method that is capable to ensure UV finiteness of a renormalizable theory, is the renormalized perturbation theory. We have a freedom in the choice of the finite parts of the counterterms (which defines the scheme), and so we have the possibility to adapt the finite parts to the environment. To compare result coming from different schemes we must ensure that the physics is the same; formally this means the requirement to keep the bare Lagrangian constant.

This line of thought can be continued and apply to the momentum dependent resummations, since formally the finite parts of the counterterms can be momentum dependent. To be consistent we must allow the appearance of momentum dependent renormalized parameters, too. Renormalizability can be maintained, if the momentum dependence is soft enough in the asymptotic momentum domain: in case of the mass term, for example, $m^{2}(k)=m_{R}^{2}+\mathscr{O}\left(k^{-\gamma}\right)$ momentum dependence is allowed for large momenta, where $\gamma>0$.

In this way we can reproduce the $2 \mathrm{PI}$ resummation with momentum dependent schemes. The naive definition, however, turn out to be non-renormalizable - just like the usual 2PI resummation. In this context the problem of renormalizability can be solved by omitting the problematic terms in the asymptotic momentum region. In this way we should abandon the complete self-energy (2PI) resummation, but we can still maintain the 2PI resummation in the infrared regime. 


\section{Acknowledgment}

The author thanks for A. Patkós, U. Reinosa and Zs. Szép for discussions. Support by the Hungarian National Research Fund OTKA (F043465), by the Hungarian Research and Technological Innovation Fund, and by the Croatian Ministry of Science, Education and Sports is gratefully acknowledged.

\section{References}

[1] L. Dolan and R. Jackiw, Phys.Rev. D 9 (1974) 3320.

[2] E. Braaten and R. Pisarski, Nucl.Phys. B 337 (1990) 569.; B 339 (1990) 662.

[3] J. Berges, AIP Conf.Proc.739:3-62,2005, hep-ph/0409233

[4] J. Collins, Renormalization (Cambridge University Press, 1984.)

[5] J. Berges, Sz. Borsanyi, U. Reinosa, J. Serreau, Phys.Rev.D71:105004 (2005) [hep-ph/0409123]; J.-P. Blaizot, E. Iancu and U. Reinosa, Nucl.Phys. A 736 (2004) 149; Phys.Lett. B 568 (2003) 016003; H. van Hees and J. Knoll, Phys.Rev. D 66 (2002) 025028; H.C. de Godoy Caldas, Phys.Rev. D 65 (2002) 065005.; H. Verschelde, Phys.Lett. B 497 (2001) 165; N. Banerjee and S. Mallik, Phys. Rev. D 43 (1991) 3368 .

[6] D. O'Connor and C.R. Stephens, Int.J.Mod.Phys. A9 (1994) 2805; Erratum-ibid. A9 (1994) 5851.

[7] A. Jakovac and Zs. Szep, Phys. Rev. D 71, 105001 (2005)

[8] J. Frenkel, A. Saa and J. Taylor, Phys.Rev. D 46 (1992) 3670; R. Parwani, Phys.Rev. D 45 (1994) 4965.

[9] A. Jakovac, in preparation. 